\title{
Errors in miRNA recognition
}

\author{
Pavel Vorozheykin \\ NSU, Novosibirsk, Russia \\ Pavel.Vorozheykin@gmail.com
}

\author{
Igor Titov \\ ICG SB RAS, Novosibirsk, Russia \\ NSU, Novosibirsk, Russia \\ Titov@bionet.nsc.ru
}

\begin{abstract}
Selecting and using the key miRNA features are crucial for the identification of miRNA sequences. These criteria may help us to take out junk data from the miRNA databases and to improve the prediction of miRNA ends in order to obtain more accurate forecasting of the seed-related functions. We consider different processes of the miRNA identification to determine their role and influence on the prediction quality.
\end{abstract}

Keywords - miRNA, pre-miRNA, miRBase, MirGeneDB, prediction method, miRNA origin, miRNA biogenesis

\section{Motivation and Aim}

MicroRNAs constitute a large class of small non-coding RNAs which clearly play an important role in the biological regulation system. Great interest of researchers led to a rapid increase of both publications and annotated miRNA sequences in the miRBase database [1]. However, only a small part of them are completely described and experimentally validated. During the process of data accumulation, the number of invalid samples is inevitably growing. To date the miRBase contains not only the false positives but lacks large number of bona fide miRNAs and pre-miRNAs [2]. So, the existing annotations and validation criterias are becoming obsolete and need to be revised and upgraded from time to time.

\section{Results}

Each step of the work with miRNAs introduces some distortions in processed data, so called natural, experimental and computational errors.

Natural errors occur when the miRNAs are cutting by the Dicer and Drosha complexes and appear as the heterogeneous miRNA ends. However, these defects can be effectively corrected by exosomes' trimming and tailing mechanisms. Such processes of miRNA maturation produce a natural noise, which (among other mechanisms) serves as an evolutionary driver of miRNA development and is involved in the creation of novel miRNAs.

Natural errors have significantly impact on the experimental errors. This type of errors is due to incorrect experimental validation of the miRNA functions. As a result, the miRNA database is updated with incorrect annotated miRNAs some of which belong to the transcriptional garbage (e.g. tRNAs). Other false-positive miRNAs demonstrate abnormal heterogeneous ends or cannot be observed in repeatable experiments at all (e.g. xenomiRs).
Finally, the natural and experimental errors are accumulated in training datasets which leading to inaccuracy of the computational methods. Existing computational and heuristic methods which predict the miRNA based on the criteria from different areas of knowledge about miRNAs. Ones predict the miRNAs by applying the evolutionary conservation of sequences and functions across the related species, the sequence and structure similarity and cluster analysis of the miRNA locations. Other methods implement machine learning algorithms (Hidden Markov Models, Support Vector Machines, Naive Bayesian Classifiers, etc.) using the context-structural characteristics of the miRNA sequences and their precursors (pri-/pre-miRNAs). To improve the method's efficiency ones add more intrinsic sequence features, such as oligonucleotide motifs, nucleotide frequencies, MFE of the precursor hairpin, conserved stems and loops, etc. Finally, the most costly and complex methods take the advantages of the next generation sequencing and experimental validation [3]. To increase the quality the prediction methods may include several criteria simultaneously [4].

We consider the contribution of these independent and combined criteria for the classification of putative miRNAs and on the prediction of miRNAs.

\section{Conclusion}

Results can be useful to adjust the methods either to predict new microRNAs or to verify already annotated ones. Also, the description of the significant miRNA features can shed light on the miRNA's evolutionary origin and on the specificities of the pri-/pre-/miRNA processing and contextstructural characteristics of sequences.

\section{REFERENCES}

[1] Kozomara A., Birgaoanu M., Griffiths-Jones S. (2019). miRBase: from microRNA sequences to function. Nucleic Acids Research. 47(D1):D155-D162.

[2] Fromm B., Billipp T., Peck L.E., et al. (2015). A uniform system for the annotation of vertebrate microRNA genes and the evolution of the human microRNAome. Annual review of genetics. 49:213-242.

[3] Kozomara A., Griffiths-Jones S. (2019). miRBase: annotating high confidence microRNAs using deep sequencing data. Nucleic Acids Research. 42(D1):D68-D73.

[4] Fromm B., Domanska D., Høye E., et al.(2020). MirGeneDB 2.0: the metazoan microRNA complement. Nucleic Acids Research. 48(D1): D132-D141. 